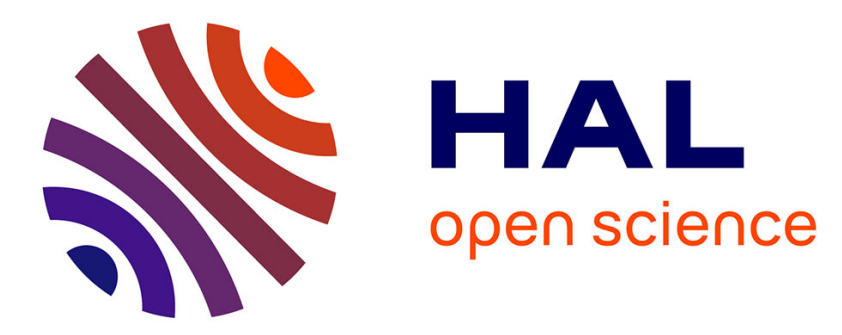

\title{
Dynamic Modeling and Controller Design of a novel aerial grasping robot
}

Zhongmou Li, Xiaoxiao Song, Vincent Begoc, Abdelhamid Chriette, Isabelle Fantoni

\section{- To cite this version:}

Zhongmou Li, Xiaoxiao Song, Vincent Begoc, Abdelhamid Chriette, Isabelle Fantoni. Dynamic Modeling and Controller Design of a novel aerial grasping robot. 23rd CISM IFToMM Symposium on Robot Design, Dynamics and Control (RoManSy 2020), Sep 2020, Sapporo, Japan. hal-02852375

\section{HAL Id: hal-02852375 \\ https://hal.science/hal-02852375}

Submitted on 11 Oct 2021

HAL is a multi-disciplinary open access archive for the deposit and dissemination of scientific research documents, whether they are published or not. The documents may come from teaching and research institutions in France or abroad, or from public or private research centers.
L'archive ouverte pluridisciplinaire HAL, est destinée au dépôt et à la diffusion de documents scientifiques de niveau recherche, publiés ou non, émanant des établissements d'enseignement et de recherche français ou étrangers, des laboratoires publics ou privés. 


\title{
Dynamic Modeling and Controller Design of a novel aerial grasping robot *
}

\author{
Zhongmou $\mathrm{Li}^{1}$, Xiaoxiao Song ${ }^{1}$, Vincent Bégoc ${ }^{1,2}$, Abdelhamid Chriette ${ }^{1}$, and \\ Isabelle Fantoni ${ }^{1}$ \\ 1 Laboratoire des Sciences du Numérique de Nantes (LS2N), \\ ECN, UMR CNRS 6004, 44321 Nantes, France \\ ${ }^{2}$ Institut catholique d'arts et métiers (Icam) \\ $\{$ Firstname. Lastname $\}$ @ls2n.fr
}

\begin{abstract}
This paper presents dynamic modelling, controller design and simulation results of a novel aerial robot dedicated to manipulation and grasping of large-size objects. This robot can be described as a flying hand composed of four fingers, four quadrotors and a body structure. The four quadrotors are arranged so as to allow the full actuation of the body structure in $\mathrm{SE}(3)$. This permits the system to maintain its position and attitude while closing the fingers despite external disturbances. The opening/closing motion of each finger is actuated by the yaw rotation of one quadrotor and transmitted through a non-backdrivable worm-gear mechanism so that the hand can produce secured grasps. We design a model predictive controller to deal with unknown mass, inertia and center of mass. The effectiveness of the controller and its robustness against external disturbances and noise are validated through several simulations using ADAMS-SIMULINK co-simulation.
\end{abstract}

Keywords: Aerial Systems · Mechanics and Control · Grasping

\section{Introduction}

Unmanned Aerial Vehicles, because of their versatility, are expected to accomplish increasingly complex tasks, such as grasping and manipulating objects [4]. Previous works embed additional mechanisms on quadrotors, such as grippers [2] and/or serial manipulators [10,15]. Adding motors reduces the energetic autonomy, furthermore, it is difficult for those approaches to deal with large objects, as a larger object often requires a larger gripper/manipulator thus reducing the payload.

Increasing the payload can be achieved using multiple quadrotors. Quadrotors can be rigidly attached to a manipulated body [8]. However, if all rotors are collinear, the obtained system remains underactuated and does not provide full-actuation in $\mathrm{SE}(3)$, such that it cannot resist any lateral disturbance without tilting. Another approach consists of using cable-suspended mechanisms actuated by multiple quadrotors $[3,7,13]$. However, even if cable robots can provide

\footnotetext{
* Supported by China Scholarship Council
} 
full actuation, they are not designed to grasp objects and the object needs to be manually attached before performing the task. Another approach to achieve full-actuation in $\mathrm{SE}(3)$, consists of using three quadrotors as rotating thrust generators, the generated forces are then transmitted to a frame, either through passive spherical joints [9], or through rigid articulated passive legs [14]. In both cases, the yaw motion of each drone cannot contribute to the manipulation task, making these robots uselessly overactuated.

In order to grasp, manipulate and transport large objects, we propose a novel robot architecture, named FlyingGripper, presented in a planar version, in a previous article [12]. The version of the $3 \mathrm{D}$ robot can be described as a flying hand composed of a body structure, four fingers and four quadrotors arranged so as to allow the full actuation of the body structure in $\mathrm{SE}(3)$ (not all rotors are collinear). This permits the system to maintain its position and attitude while closing the fingers despite any external disturbance. The yaw motion of each quadrotor is used to actuate the opening/closing motion of each finger.

A model predictive controller is designed to track a trajectory considering varying dynamic parameters such as model mismatch. Therefore, control input is calculated through a control allocation algorithm. A co-simulation shows the ability of the FlyingGripper to grasp, transport and place a large object in the presence of noise and disturbances. Finally, conclusion and future work are given.

\section{Presentation of the FlyingGripper}

The FlyingGripper is composed of four quadrotors, four fingers and a body structure Fig. 1. Each finger has two phalanges and is underactuated so that it can adapt to the size and shape of the object and deal with position uncertainty. Each quadrotor is linked to the body structure through a passive revolute joint so that it can rotate along its yaw axis with respect to the body structure. This yaw motion is transmitted through a worm-gear mechanism to an actuation bar to open and close the finger Fig. 1(b). This worm-gear mechanism is chosen to be non-backdrivable to produce form-closure grasps [5]. This stability criteria yields to secured grasps which do not rely on the capability of actuators nor on friction between phalanges and the object, and furthermore do not require any additional energy for grasping during the flight. More details on the closing sequence of the FlyingGripper can be found in [12].

Quadrotors are arranged so as to provide full-actuation of the robot in $\operatorname{SE}(3)$, i.e. yaw axes of quadrotors are not colinear but tilted along the $x$ and $y$ directions of the frame $\mathscr{F}_{G}$ attached to the body structure Fig. 1. The manipulability of our $3 \mathrm{D}$ verison robot is not analyzed in this paper, since a methodology was presented in [12] to optimize the design of a planar version of the Flying Gripper. However, we present here full manipulation of the 3D version of the FlyingGripper, which permits to maintain its position and attitude during grasping despite external disturbances. 


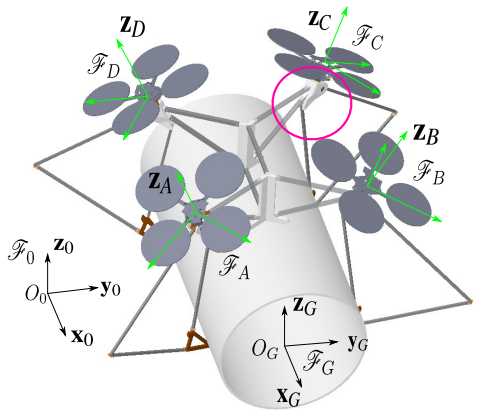

(a)

Fig. 1: (a) CAD view of the FlyingGripper composed of four quadrotors, four underactuated fingers and a body structure, (b) Opening/closing motion of each finger does not require any additional actuator since it uses the yaw rotation of each quadrotor transmitted, through a worm-gear mechanism, to the finger's actuation bar.

\section{Static and dynamic modeling of the FlyingGripper}

Let $\mathbf{q}=\left[\begin{array}{lll}\boldsymbol{\xi}^{T} & \boldsymbol{\eta}^{T} \boldsymbol{\gamma}^{T}\end{array}\right]^{T}$ be the vector of generalized coordinates which fully describe the configuration of the robot, where:

$-\boldsymbol{\xi}=\left[\begin{array}{ll}x y & z\end{array}\right]^{T}$ is the position vector of the origin of the body frame $\mathscr{F}_{G}$ expressed in the world frame $\mathscr{F}_{0}$,

$-\boldsymbol{\eta}=\left[\begin{array}{lll}\phi & \theta & \psi\end{array}\right]^{T}$ is the vector of Euler angles denoting the orientation of $\mathscr{F}_{G}$ with respect to $\mathscr{F}_{0}$,

$-\gamma=\left[\begin{array}{llll}\gamma_{A} & \gamma_{B} & \gamma_{C} & \gamma_{D}\end{array}\right]^{T}$, with $\gamma_{i}$ the yaw rotation angle of the $i^{\text {th }}$ quadrotor expressed in the quadrotor's local frame $\mathscr{F}_{i}(i=A, B, C, D)$.

The following assumptions are made in order to simplify the expressions of the dynamic model: $\mathscr{F}_{G}$ coincides with the Center of Mass (CoM) of the whole system (the robot and the object) and the origin of $\mathscr{F}_{i}=\left\{O_{i}, \mathbf{x}_{i}, \mathbf{y}_{i}, \mathbf{z}_{i}\right\}$, coincides with the CoM of quadrotor $i$.

The static equilibrium of the FlyingGripper is given by:

$$
\left\{\begin{array}{l}
\mathbf{f}+\sum_{i=A}^{D}{ }^{G} \mathbf{R}_{i} f_{z_{i}} \mathbf{z}_{i}=\mathbf{0} \\
\boldsymbol{\tau}+\sum_{i=A}^{D}\left({ }^{G} \widehat{\mathbf{r}}_{i}{ }^{G} \mathbf{R}_{i} f_{z_{i}} \mathbf{z}_{i}+{ }^{G} \mathbf{R}_{i} \boldsymbol{\tau}_{i}\right)=\mathbf{0}
\end{array}\right.
$$

where $\mathbf{f}$ and $\boldsymbol{\tau}$ are respectively the external force and torque vectors exerted on the body structure expressed in $\mathscr{F}_{G}, f_{z_{i}}$ and $\boldsymbol{\tau}_{i}$ are respectively the thrust force and the torque vector exerted by the $i$ th quadrotor expressed in the local frame $\mathscr{F}_{i},{ }^{G} \mathbf{R}_{i}$ is the rotation matrix of $\mathscr{F}_{i}$ expressed in $\mathscr{F}_{G},{ }^{G} \mathbf{r}_{i}$ is the coordinate vector of $O_{i}$ expressed in $\mathscr{F}_{G}$ and ${ }^{G} \widehat{\mathbf{r}}_{i}$ is the skew-symmetric matrix of ${ }^{G} \mathbf{r}_{i}$.

Introducing $\mathbf{w}=\left[\mathbf{f}^{T} \boldsymbol{\tau}^{T}\right]^{T} \in \mathbb{R}^{6 \times 1}$ the external wrench applied on the body structure and $\mathbf{w}_{q}=\left[\mathbf{w}_{A}^{T} \mathbf{w}_{B}^{T} \mathbf{w}_{C}^{T} \mathbf{w}_{D}^{T}\right]^{T} \in \mathbb{R}^{16 \times 1}$ the concatenation of wrenches applied by the four quadrotors on the body structure, with $\mathbf{w}_{i}=\left[f_{z_{i}} \boldsymbol{\tau}_{i}^{T}\right]^{T} \in$ 
$\mathbb{R}^{4 \times 1}$, the equilibrium expression(1) can be written in the following form:

$$
\mathbf{w}+\mathbf{W} \mathbf{w}_{q}=\mathbf{0}
$$

where $\mathbf{W}=\left[\mathbf{W}_{A}^{r} i \mathbf{W}_{B}^{r} \vdots \mathbf{W}_{C}^{r} \vdots \mathbf{W}_{D}^{r}\right] \in \mathbb{R}^{6 \times 16}$ is the wrench matrix mapping the quadrotors wrenches in the body frame $\mathscr{F}_{G} . \mathbf{W}_{i}^{r} \in \mathbb{R}^{6 \times 4}$ is defined as

$$
\mathbf{W}_{i}^{r}=\left[\begin{array}{c:c}
{ }^{G} \mathbf{R}_{i} \mathbf{z}_{i} & \mathbf{0}_{3 \times 3} \\
\hdashline \widehat{\widehat{r}}_{i}{ }^{G} \mathbf{R}_{i} \mathbf{z}_{i} & { }^{G} \mathbf{R}_{i}
\end{array}\right] .
$$

The dynamic modeling of the whole system (robot, fingers and object) is developed considering the whole system as a rigid body. The dynamics of the fingers during grasping or releasing is also neglected as the fingers are assumed to close/open slowly. Indeed, we assume that the object, once grasped, has no motion with respect to the body frame. Therefore, the dynamic model is developed by applying Newton-Euler method:

$$
\left[\begin{array}{cc}
m \mathbf{I}_{3} & \mathbf{0}_{3} \\
\mathbf{0}_{3} & \mathbf{I}_{m}
\end{array}\right]\left[\begin{array}{c}
\ddot{\boldsymbol{\xi}} \\
\dot{\boldsymbol{\omega}}
\end{array}\right]+\left[\begin{array}{c}
m \mathbf{g} \\
\boldsymbol{\omega} \times \mathbf{I}_{m} \boldsymbol{\omega}
\end{array}\right]=\left[\begin{array}{cc}
{ }^{0} \mathbf{R}_{G} & \mathbf{0} \\
\mathbf{0} & \mathbf{I}_{3}
\end{array}\right] \mathbf{w}
$$

where $m$ is the mass and $\mathbf{I}_{m}$ is the inertia matrix of the whole manipulator system comprising the quadrotors and the object, $\boldsymbol{\omega}$ is the angular velocity of the robot expressed in $\mathscr{F}_{G}$ and ${ }^{0} \mathbf{R}_{G}$ is the rotation matrix of $\mathscr{F}_{G}$ relative to $\mathscr{F}_{0}$.

\section{Controller design}

The controller designed for our robot has two objectives: (1) a model predictive controller enables the robot to move from an initial to a final position following a referenc trajectory; (2) a control allocation algorithm [11] is developed to ensures the closing/opening of fingers while tracking the trajectory.

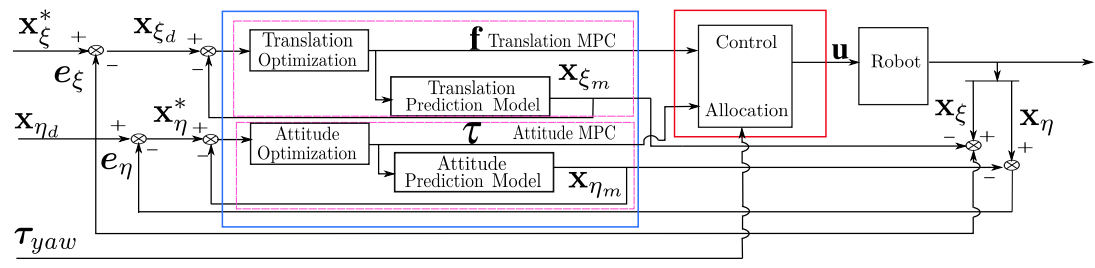

Fig. 2: General control scheme of FlyingGripper.

\subsection{Model Predictive Control for tracking a trajectory}

For our robot, variations of dynamic parameters caused by holding the object are considered as model mismatch and uncertainty. Then, a model predictive controller based on [1] is developed to track the trajectory, dealing with the varying dynamic parameters, in presence of disturbances and noise.

More precisely, Translation MPC and Attitude MPC (Fig. 2) calculate the required forces $\mathbf{f}$ and torques $\boldsymbol{\tau}$ in order to track the desired trajectory. In the Translation MPC, the input $\mathbf{f}$ can be obtained by solving the optimization problem as follows:

$$
\underset{\widetilde{U}_{\xi}}{\operatorname{minimize}} \sum_{k=n_{\xi}+1}^{n_{\xi}+N_{p}}\left[\mathbf{x}_{\xi_{d}}(k)-\mathbf{x}_{\xi_{m}}(k)\right]^{T} \mathbf{Q}_{\xi}(k)\left[\mathbf{x}_{\xi_{d}}(k)-\mathbf{x}_{\xi_{m}}(k)\right]
$$




$$
\begin{aligned}
\mathbf{x}_{\xi_{m}}(k) & =\mathbf{A}_{\xi} \mathbf{x}_{\xi_{m}}(k-1)+\mathbf{B}_{\xi} \mathbf{f}(k-1)+\mathbf{g}_{\xi} \\
\text { subject to: } \quad \boldsymbol{e}_{\xi}(k) & =\mathbf{x}_{\xi}(k)-\mathbf{x}_{\xi_{m}}(k) \\
\mathbf{x}_{\xi_{d}}(k) & =\mathbf{x}_{\xi}^{*}(k)-\boldsymbol{e}_{\xi}(k)
\end{aligned}
$$

where $\widetilde{U}_{\xi}=\left\{\mathbf{f}\left(n_{\xi}\right), \mathbf{f}\left(n_{\xi}+1\right), \ldots, \mathbf{f}(k), \ldots, \mathbf{f}\left(n_{\xi}+N_{c}-1\right)\right\}$, while $\mathbf{x}_{\xi}=\left[\boldsymbol{\xi}^{T} \dot{\boldsymbol{\xi}}^{T}\right]^{T}$ is the translation state variable, $\mathbf{x}_{\xi}^{*}(k)$ is the reference translation trajectory. The prediction model in Eq. (6) is linearized from Eq. (4) at the horizontal attitude, $\mathbf{x}_{\xi_{m}}$ is output of the prediction model to predict the robot translation state. $\boldsymbol{e}_{\xi}$ is the error between the state $\mathbf{x}_{\xi}$ and the prediction $\mathbf{x}_{\xi_{m}}$, while $\mathbf{x}_{\xi_{d}}$ is the correction of the reference trajectory $\mathbf{x}_{\xi}^{*}(k)$ with the error $\boldsymbol{e}_{\xi}$. Noticing that $\mathbf{N}_{p}$ is the prediction horizon, $\mathbf{N}_{c}$ is the the control horizon and $\mathbf{Q}_{\xi}$ is the weighting matrix.

In terms of the Attitude MPC, the state variable is $\mathbf{x}_{\eta}=\left[\boldsymbol{\eta}^{T} \dot{\boldsymbol{\eta}}^{T}\right]^{T}$. The attitude prediction model is based on the linearization of the dynamic model in Eq. (4) at the horizontal attitude to obtain the required torque $\tau$.

\subsection{A control allocation algorithm ensuring closing/opening the fingers}

For our robot, the control input of each quadrotor is the motor speed that can be linked to the quadrotor wrench using the following linear relation [6]:

$$
\mathbf{w}_{i}=\Gamma_{i} \mathbf{u}_{i},
$$

where $\boldsymbol{\Gamma}_{i} \in \mathbb{R}^{4 \times 4}$ is an invertible matrix whose components are given from aerodynamic coefficients and geometric parameters, $\mathbf{u}_{i}=\left[\begin{array}{llll}\varpi_{i 1}^{2} & \varpi_{i 2}^{2} \varpi_{i 3}^{2} \varpi_{i 4}^{2}\end{array}\right]^{T}$, with $\varpi_{i j}$ being the rotational speed of the $j$ th rotor of the $i$ th quadrotor.

Introducing $\mathbf{u}=\left[\begin{array}{l}\mathbf{u}_{A}^{T} \mathbf{u}_{B}^{T} \mathbf{u}_{C}^{T} \mathbf{u}_{D}^{T}\end{array}\right]^{T} \in \mathbb{R}^{16 \times 1}$ the control input of the robot, Eq. (2) can be rewritten as:

$$
\mathbf{w}+\mathbf{A u}=\mathbf{0}
$$

with $\mathbf{A}=\mathbf{W} \boldsymbol{\Gamma} \in \mathbb{R}^{6 \times 16}$, with $\boldsymbol{\Gamma}=\operatorname{diag}\left(\boldsymbol{\Gamma}_{i}\right)$, is a matrix that maps $\mathbf{u} \in \mathbb{R}^{16 \times 1}$ onto the wrench $\mathbf{w} \in \mathbb{R}^{6 \times 1}$.

The control input $\mathbf{u}$ must be chosen so as to satisfy Eq (10) and so that each quadrotor exerts a yaw torque $\tau_{z_{i}}$ on the worm screw in order to open or close the corresponding finger. Let introduce $\tau_{y a w}=\left[\begin{array}{llll}\tau_{z_{A}} & \tau_{z_{B}} & \tau_{z_{C}} & \tau_{z_{D}}\end{array}\right]^{T}$ the vector of yaw torques exerted by the four quadrotors. It can be expressed from the input vector using $\boldsymbol{\tau}_{\text {yaw }}=\mathbf{C u}$, where $\mathbf{C}=\operatorname{diag}\left(\mathbf{c}_{i}\right)$ with $\mathbf{c}_{i}$ being the last row of matrix $\boldsymbol{\Gamma}_{i}$ in Eq. (9). Thus, the control input $\mathbf{u}$ can be obtained as

$$
\left[\begin{array}{c}
\mathbf{w} \\
\boldsymbol{\tau}_{\text {yaw }}
\end{array}\right]=\left[\begin{array}{c}
-\mathbf{A} \\
\mathbf{C}
\end{array}\right] \mathbf{u}=\mathbf{H u} .
$$

Since the dimension of $\mathbf{u} \in \mathbb{R}^{16 \times 1}$ is greater than that of $\left[\mathbf{w}^{T} \boldsymbol{\tau}_{y a w}^{T}\right]^{T} \in \mathbb{R}^{10 \times 1}$, the system is overactuated and there exists an infinity of solutions for $\mathbf{u}$ to Eq. (11). The control input $\mathbf{u}$ is restricted by the limits of the rotors and must satisfy $\underline{\mathbf{u}} \leq \mathbf{u} \leq \overline{\mathbf{u}}$, where $\underline{\mathbf{u}}$ and $\overline{\mathbf{u}}$ are resp. the lower and upper bounds to the rotor square speeds. In order to increase the possibility of finding solutions respecting the motor speed limits, we apply the algorithm in [11] to compute $\mathbf{u}$ :

$$
\mathbf{u}=\mathbf{u}_{m}+\mathbf{H}^{+}\left(\left[\begin{array}{c}
\mathbf{w} \\
\boldsymbol{\tau}_{\text {yaw }}
\end{array}\right]-\mathbf{H u}_{m}\right)
$$


where $\mathbf{u}_{m}=(\underline{\mathbf{u}}+\overline{\mathbf{u}}) / 2$ and $\mathbf{H}^{+}$is the Moore-Penrose pseudoinverse of $\mathbf{H}$.

\section{Simulation results}

A simulator was designed using MATLAB-SIMULINK and ADAMS co-simulation. The dynamic of the flying robot was simulated by ADAMS, while the controller was implemented on MATLAB-SIMULINK. A white noise of amplitude $\pm 10^{-3}$ ( $\mathrm{m}$ or rad) was introduced to each measurement of $\mathbf{q}$ and a varying disturbance force $\mathbf{f}_{d}$ was applied to the CoM of the robot in the ADAMS model.

\subsection{Robot grasping a $1 \mathrm{~kg}$ object with disturbances}

In this first simulation, the robot is required to perform a downward trajectory and the close its fingers while maintaining its position above the object. The results are shown in Fig.3, where we can see that the robot is able to complete the task with the controller under disturbances and sensor noise. Table 1 gives the mean of absolute value of the tracking error for each coordinate for different amplitudes of the disturbance force.

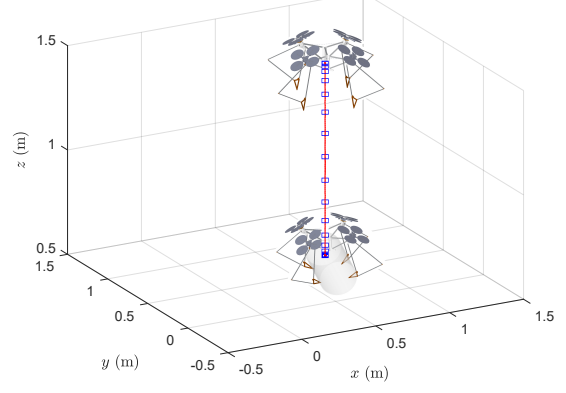

(a) Robot's trajectory

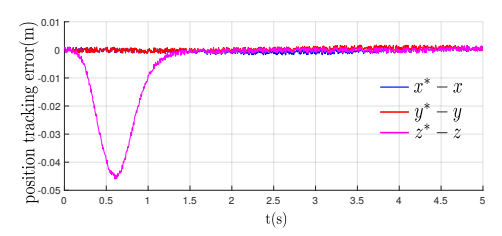

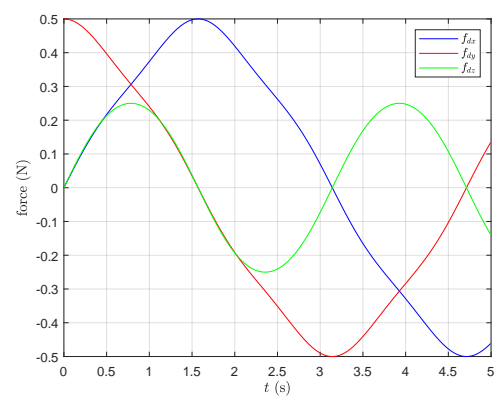

(b) Disturbance forces

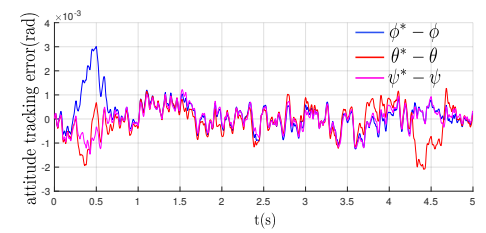

(c) Tracking error

Fig. 3: FlyingGripper performs a downward trajectory $(0-2 \mathrm{~s})$ and then closes the fingers while maintaining its position and attitude $(2 \mathrm{~s}-5 \mathrm{~s})$ under noise and a disturbance force

\subsection{Robot grasping, transporting and placing a $1 \mathrm{~kg}$ object}

In reality, the robot is always required to accomplish more complex tasks, for instance to grasp, manipulate and place an object. Thus, in this simulation, the robot is supposed to approach the object $(1 \mathrm{~kg})$, close the fingers, transport the object and place the object under disturbances (Fig.3) and sensor noise. The simulation results are shown in Fig.4. 
Table 1: Mean of absolute value of the tracking error of each coordinate

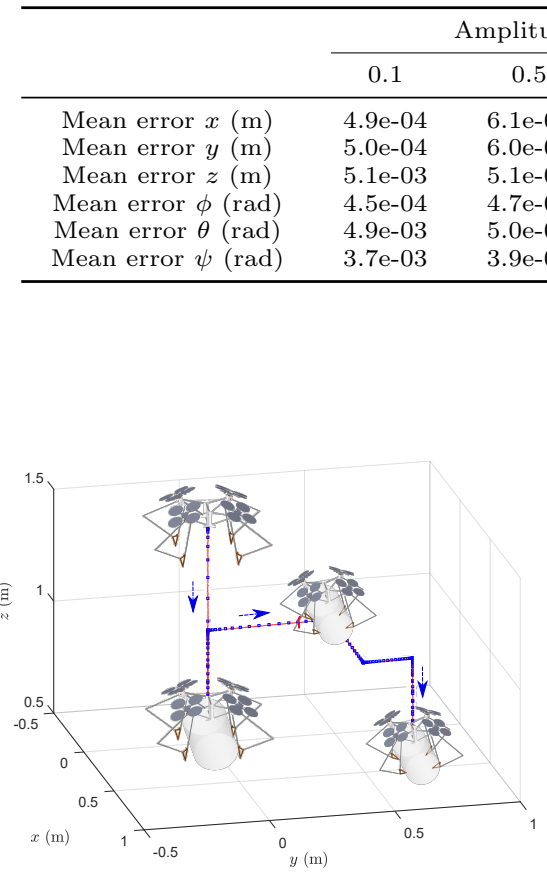

(a) Robot's trajectory

\begin{tabular}{cccc}
\multicolumn{4}{c}{ mplitude of force disturbances $(\mathrm{N})$} \\
\hline 0.5 & 1 & 3 & 5 \\
\hline $6.1 \mathrm{e}-04$ & $9.7 \mathrm{e}-04$ & $2.8 \mathrm{e}-03$ & $6.3 \mathrm{e}-03$ \\
$6.0 \mathrm{e}-04$ & $9.5 \mathrm{e}-04$ & $2.9 \mathrm{e}-03$ & $6.0 \mathrm{e}-03$ \\
$5.1 \mathrm{e}-03$ & $5.2 \mathrm{e}-03$ & $8.4 \mathrm{e}-03$ & $2.2 \mathrm{e}-02$ \\
$4.7 \mathrm{e}-04$ & $6.0 \mathrm{e}-04$ & $1.6 \mathrm{e}-03$ & $8.3 \mathrm{e}-03$ \\
$5.0 \mathrm{e}-03$ & $5.9 \mathrm{e}-04$ & $2.6 \mathrm{e}-03$ & $3.2 \mathrm{e}-03$ \\
$3.9 \mathrm{e}-03$ & $3.9 \mathrm{e}-04$ & $4.0 \mathrm{e}-04$ & $5.1 \mathrm{e}-04$ \\
\hline
\end{tabular}
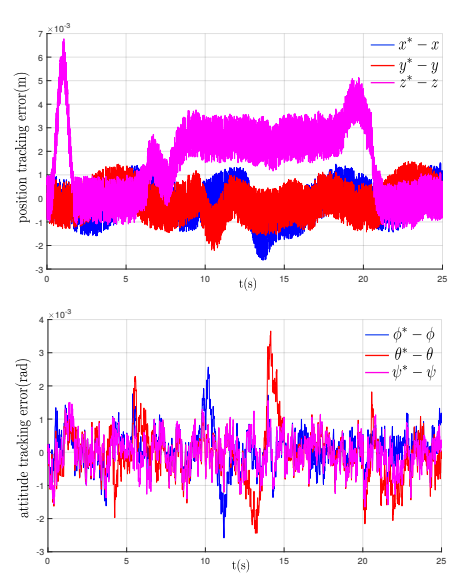

(b) Tracking error

Fig. 4: FlyingGripper performs a downward trajectory approaching the object in $0-3 \mathrm{~s}$, closing the fingers in $3 \mathrm{~s}-6 \mathrm{~s}$, transporting the object in $6 \mathrm{~s}-21 \mathrm{~s}$ and placing the object in $21 \mathrm{~s}-25 \mathrm{~s}$ under disturbances (Fig.3) and sensor noise.

This result illustrates that the robot is capable of grasping the object and dealing with the variation of dynamics caused by the object in the manipulation. It demonstrates the effectiveness of the designed controller and its robustness against noise and disturbance. A video of this co-simulation is available online at ${ }^{1}$.

\section{Conclusion and future work}

In this paper, we have built the static and dynamic model for the novel aerial grasping robot. The developed MPC controller, based on the dynamic model, enables the robot to track a reference trajectory, dealing with varying dynamic parameters, such as unknown mass, inertia and CoM. The control allocation algorithm ensures the closing/opening of the fingers while tracking the trajectory. Simulations results validate effectiveness of the controller and its robustness against external disturbances and noise. The next step is to build the prototype and test the controller in real-time experiments.

\footnotetext{
${ }^{1}$ https://youtu.be/ziYE_SF3t4c
} 


\section{References}

1. Allibert, G., Courtial, E., Chaumette, F.: Predictive control for constrained imagebased visual servoing. IEEE Transactions on Robotics 26(5), 933-939 (2010)

2. Car, M., Ivanovic, A., Orsag, M., Bogdan, S.: Impedance based force control for aerial robot peg-in-hole insertion tasks. In: 2018 IEEE/RSJ IROS. pp. 6734-6739 (2018)

3. Erskine, J., Chriette, A., Caro, S.: Wrench Analysis of Cable-Suspended Parallel Robots Actuated by Quadrotor Unmanned Aerial Vehicles. Journal of Mechanisms and Robotics 11(2) (2019)

4. Khamseh, H.B., Janabi-Sharifi, F., Abdessameud, A.: Aerial manipulation - a literature survey. Robotics and Autonomous Systems 107, 221-235 (2018)

5. Krut, S., Bégoc, V., Dombre, E., Pierrot, F.: Extension of the form-closure property to underactuated hands. IEEE Transactions on Robotics 26(5), 853-866 (2010)

6. Mahony, R., Kumar, V., Corke, P.: Multirotor aerial vehicles: Modeling, estimation, and control of quadrotor. IEEE Robotics and Automation mag. 19(3), 20-32 (2012)

7. Manubens, M., Devaurs, D., Ros, L., Cortés, J.: Motion planning for 6-D manipulation with aerial towed-cable systems. In: Robotics: Science and Systems. Berlin, Germany (May, 2013)

8. Michael, N., Fink, J., Kumar, V.: Cooperative manipulation and transportation with aerial robots. Autonomous Robots 30(1), 73-86 (2011)

9. Nguyen, H.N., Park, S., Park, J., Lee, D.: A novel robotic platform for aerial manipulation using quadrotors as rotating thrust generators. IEEE Transactions on Robotics 34(2), 353-369 (2018)

10. Orsag, M., Korpela, C., Bogdan, S., Oh, P.: Dexterous aerial robots-mobile manipulation using unmanned aerial systems. IEEE Transactions on Robotics 33(6), 1453-1466 (2017)

11. Pott, A., Bruckmann, T., Mikelsons, L.: Closed-form force distribution for parallel wire robots. In: Computational Kinematics, pp. 25-34. Springer (2009)

12. Saint-Sevin, M., Bégoc, V., Briot, S., Chriette, A., Fantoni, I.: Design and optimization of a multi-drone robot for grasping and manipulation of large size objects. In: Proceedings of the 22nd CISM IFToMM Symposium, pp. 458-465 (2018)

13. Sanalitro, D., Savino, H.J., Tognon, M., Cortés, J., Franchi, A.: Full-pose manipulation control of a cable-suspended load with multiple uavs under uncertainties. IEEE Robotics and Automation Letters (2020)

14. Six, D., Briot, S., Chriette, A., Martinet, P.: The kinematics, dynamics and control of a flying parallel robot with three quadrotors. IEEE Robotics and Automation Letters 3(1), 559-566 (2018)

15. Suarez, A., Soria, P.R., Heredia, G., Arrue, B.C., Ollero, A.: Anthropomorphic, compliant and lightweight dual arm system for aerial manipulation. In: IEEE/RSJ IROS. pp. 992-997 (2017) 